\title{
Analyse d'une catégorie juridique récente : le mineur étranger non accompagné, séparé ou isolé
}

Analysing a Recent Legal Category: Unaccompanied or Separated Children

Análisis de una categoría jurídica reciente: el menor extranjero no acompañado o separado

Daniel Senovilla Hernández

\section{CpenEdition}

Journals

Édition électronique

URL : https://journals.openedition.org/remi/6732

DOI : $10.4000 /$ remi.6732

ISSN : $1777-5418$

Éditeur

Université de Poitiers

Édition imprimée

Date de publication : 1 mars 2014

Pagination : 17-34

ISBN : 979-10-90426-21-4

ISSN : 0765-0752

Référence électronique

Daniel Senovilla Hernández, « Analyse d'une catégorie juridique récente : le mineur étranger non accompagné, séparé ou isolé », Revue européenne des migrations internationales [En ligne], vol. $30-n^{\circ} 1$ I 2014, mis en ligne le 01 mars 2017, consulté le 14 avril 2022. URL : http://journals.openedition.org/ remi/6732; DOl : https://doi.org/10.4000/remi.6732 


\section{Analyse d'une catégorie juridique récente : le mineur étranger non accompagné, séparé ou isolé}

\section{Daniel Senovilla Hernández ${ }^{1}$}

La migration de personnes mineures ${ }^{2}$ existe depuis plusieurs siècles; elle est souvent effectuée dans un cadre familial, mais aussi de façon indépendante. Ce n'est cependant qu'au cours de la dernière décennie du XXe siècle que le phénomène acquière une dimension propre (déliée des migrations de personnes adultes) et commence à attirer l'attention des acteurs institutionnels et associatifs et, progressivement, du monde académique (Bhabha, 2010 : 92).

Dans le contexte de I'Union européenne, les premiers cas médiatisés de migrations de mineurs soulignent l'existence d'un vide juridique. Ainsi, dès le début des années 1990, I'Allemagne, le Royaume-Uni, la Belgique, la France, et bien d'autres États membres, enregistrent des demandes d'asile de personnes de moins de dix-huit ans qui se présentent à leurs frontières " non accompagnées " de leurs parents ou de représentants légaux. Quelques années plus tard, I'Espagne et I'Italie doivent faire face à un flux croissant de mineurs seuls venus du Maghreb et d'Afrique de l'Ouest. Chaque État est obligé d'improviser une réponse juridique pour faire face à la présence de ce nouvel " acteur migratoire" (Suarez, $2004: 40)$. Les réponses apportées sont différentes au sein de ces États. Certains choisissent d'intégrer cette nouvelle catégorie de migrants dans leur droit commun, d'autres élaborent une législation spécifique ou encore optent pour une combinaison de droit commun et de normes juridiques spécifiques.

Parallèlement, les institutions communautaires s'intéressent timidement à cette migration. En 1997, le Conseil de I'Union européenne émet une résolution,

1 Chercheur post-doctoral, Université de Poitiers/CNRS, MIGRINTER (UMR 7301),

MSHS, Bâtiment A5, 5 rue Théodore Lefebvre, TSA 21103, 86073 Poitiers cedex 9; daniel. senovilla@univ-poitiers.fr

2 Nous utilisons dans ce texte une définition " juridique " de la minorité, référencée concrètement sous I'âge qui marque la fin de cette étape (dix-huit ans) selon les postulats de la Convention de Nations Unies relative aux Droits de I'Enfant, texte adopté et ouvert à la signature, ratification et adhésion par l'Assemblée générale dans sa résolution 44/25 du 20 novembre 1989. L'article 1 définit un " enfant comme tout être humain âgé de moins de dix-huit ans, sauf si la majorité est atteinte plus tôt en vertu de la législation qui lui est applicable ». 
instrument juridique non contraignant, concernant les " mineurs non accompagnés ressortissants de pays tiers ". À partir de l'expression de ce premier terme et de sa définition, le corpus juridique communautaire relatif à la réglementation de l'asile et de l'immigration s'enrichit de quelques références isolées aux " mineurs non accompagnés ", dont le contenu souligne bien souvent la volonté d'assouplir les normes sécuritaires prévues pour les migrants adultes pour traiter de cette nouvelle catégorie de migrants ${ }^{3}$.

À l'échelle des Nations Unies, I'applicabilité de la Convention Internationale relative aux Droits de I'Enfant (CIDE) aux mineurs en situation de migration apparaît clairement à la lecture de l'article $2^{4}$. Toutefois, I'interprétation de ses postulats, en particulier du principe de l'intérêt supérieur de l'enfant, et son application à la situation des mineurs migrants sont demeurées amplement indéfinies. Il faudra attendre septembre 2005 pour que le Comité des Droits de I'Enfant des Nations Unies publie un instrument de " droit souple $"^{5}$ proposant une interprétation de la CIDE et de son application à la situation des enfants « non accompagnés " et " séparés " en dehors de leur pays d'origine ${ }^{6}$. Ce document a été postérieurement développé et complété dans le cadre de rapports issus d'agences internationales ou d'organismes supranationaux (comme le Conseil de I'Europe, le Haut Commissariat pour les Réfugiés et I'UNICEF) et eux-mêmes commentés par un nombre relativement réduit de contributions académiques (entre autres, Bhabha, 2007 ; Farrugia etTouzenis, 2010 ; Senovilla Hernández, 2010).

Environ vingt ans après l'émergence de cette thématique, il paraît opportun de réaliser un bilan analytique de la catégorie juridique construite par les législateurs aux échelles internationale (Nations Unies et Union européenne) et nationale, et de la confronter aux situations de terrain, notamment observées dans le cadre d'enquêtes réalisées dans quatre États membres de l'Union européenne (France, Belgique, Espagne et Italie) entre 2011 et 2013 (Senovilla Hernández, 2013).

\section{Termes, définitions et éléments composant la catégorie juridique}

Si la définition de certaines catégories de personnes étrangères peut être préétablie par le droit international (l'exemple le plus paradigmatique étant la définition du réfugié), à ce jour aucun instrument juridique supranational n'impose de définition du mineur en situation de migration.

\footnotetext{
3 Marie Diop propose une analyse détaillée de la législation communautaire concernant les mineurs non accompagnés et de sa conformité avec les postulats de la Convention Internationale relative aux Droits de I'Enfant (Diop, 2009).

4 Article 2 de la CIDE : « Les États parties s'engagent à respecter les droits qui sont énoncés dans la présente Convention et à les garantir à tout enfant relevant de leur juridiction, sans distinction aucune, indépendamment de toute considération de race, de couleur, de sexe, de langue, de religion, d'opinion politique ou autre de l'enfant ou de ses parents ou représentants légaux, de leur origine nationale, ethnique ou sociale, de leur situation de fortune, de leur incapacité, de leur naissance ou de toute autre situation ".

5 Pour une définition de la notion de droit souple, ou soft law en terminologie anglosaxonne, voir Bousta (2010: 56).

6 Comité des Droits de I'Enfant des Nations Unies (2005) Observation générale $n^{\circ} 6$ sur le traitement des enfants non accompagnés et des enfants séparés en dehors de leur pays d'origine, CRC/GC/2005/6, 1er septembre.
} 
Le terme le plus utilisé dans le contexte européen et mondial est celui de " mineur étranger non accompagné ". Ce terme fait l'objet de toute une série de déclinaisons se différenciant selon l'organisation ou l'institution émettrice. À l'échelle des Nations Unies, un enfant non accompagné est considéré comme un " enfant de moins de dix-huit ans qui a été séparé de ses deux parents et $\mathrm{d}^{\prime}$ autres membres proches de sa famille et $\mathrm{n}^{\prime}$ est pas pris en charge par un adulte investi de cette responsabilité par la Loi et la coutume ${ }^{7}$. Les institutions de I'Union européenne utilisent ce même terme qu'elles définissent comme " un ressortissant d'un pays tiers âgé de moins de dix-huit ans qui entre ou séjourne sur le territoire d'un État membre sans être accompagné d'un adulte qui soit responsable de lui, de par la loi ou la coutume, et tant qu'il n'est pas effectivement pris en charge par une telle personne ${ }^{8}$. De leur côté, la plupart des États d'Europe utilise la même terminologie, bien qu'il soit rare de trouver une définition précise dans un instrument de rang législatif9.

Le terme " mineur séparé " entre également dans la terminologie développée pour décrire cette migration même s'il n'est nullement usité au niveau législatif. Son émergence s'inscrit dans une démarche de complémentarité à celui de " mineur non accompagné " en prétendant établir une définition moins réductrice des situations que peuvent vivre tous les mineurs migrant en l'absence des titulaires de l'autorité parentale. Ainsi, le Comité des Droits de I'Enfant des Nations Unies, en complément du " mineur non accompagné, définit le " mineur séparé " comme un enfant "qui a été séparé de ses deux parents ou des personnes qui en avaient la charge à titre principal auparavant en vertu de la loi ou de la coutume, mais pas nécessairement d'autres membres de sa famille. Un enfant séparé peut donc être accompagné par un autre membre adulte de sa famille ${ }^{10}$. Si les Nations Unies s'appuient sur ces deux termes, de son côté, le Programme sur les enfants séparés en Europe ${ }^{11}$ prône I'utilisation exclusive de l'expression " enfant séparé ", estimant qu'elle " définit mieux le problème fondamental auquel ces enfants seront confrontés ${ }^{12}$, en référence à I'absence de responsables parentaux du mineur.

7 Comité des Droits de l'Enfant des Nations Unies (2005) Observation générale $n^{\circ} 6$, op. cit., paragraphe 7.

8 Article 1 de la Résolution du Conseil de I'Union européenne du 26 juin 1997 concernant les mineurs non accompagnés ressortissants de pays tiers (97/C 221/03). Une définition similaire est reproduite dans les Directives et Règlements communautaires régissant l'immigration et l'asile qui ont été approuvés par la suite.

9 La Belgique est I'un des seuls États qui aient prévu une définition de la figure du mineur étranger non accompagné dans une norme de rang légal. Il s'agit de la Loi-programme du 24 décembre 2012, Titre XIII, Chapitre VI relatif à la tutelle des mineurs étrangers non accompagnés, article (479) 5.

10 Comité des Droits de I'Enfant des Nations Unies (2005) Observation générale $n^{\circ} 6$, op. cit., paragraphe 8.

11 Le SCEP (Separated Children in Europe Programme dans sa dénomination en langue anglaise) constituait à l'origine une initiative jointe du Haut Commissariat des Nations Unies pour les Réfugiés et de I'organisation internationale Save the Children. Actuellement, le réseau est coordonné par I'association Défense de I'Enfance Internationale aux Pays-Bas.

12 Save the Children (2004) Programme des Enfants Séparés en Europe, Déclaration de Bonne Pratique, $3^{\text {ème }}$ édition, UNHCR, $49 \mathrm{p}$. 
En ce qui concerne l'expression " mineur isolé étranger ", il s'agit d'une spécificité proprement française. Cependant, si ce terme est largement adopté par les institutions et les praticiens, il demeure absent des lois et codes qui sont appliqués à cette population : sur la législation de protection de l'enfance, le Code de l'Action Sociale et des Familles (CASF) et le Code Civil ; sur la législation des étrangers, le Code de l'entrée et séjour des étrangers et du droit d'asile (CESEDA). Curieusement, ce dernier fait plutôt référence au " mineur non accompagné d'un représentant légal ${ }^{13}$. Plus récemment, en 2013, une proposition de loi fait référence aux " mineurs isolés étrangers " sans pour autant en proposer une définition ${ }^{14}$. L'absence de définition législative du terme n'empêche toutefois pas son utilisation dans des normes réglementaires comme l'illustre la récente Circulaire Taubira du 31 mai $2013^{15}$. Dans le cadre français, l'ajout du terme " isolé " se justifie par la supposée situation d'absence des représentants légaux du mineur et des conséquences liées à son incapacité juridique (Masson, 2010 : 115) et la signification ne diffère pas fondamentalement de celles apportées par les autres termes préalablement cités.

En dehors des nuances décrites dans les paragraphes précédents, les trois termes nous renvoient à une même catégorie juridique composée de trois éléments : la minorité d'âge ; la condition d'étranger ; la potentielle situation de danger ou de délaissement liée à l'absence des parents ou des représentants légaux du mineur.

Indépendamment de l'âge frontière choisi (dix-huit ans en France et dans la plupart des pays du monde), la notion de minorité civile est simplement définie par rapport à celle de majorité : " âge déterminé par la loi à partir duquel l'individu réputé capable de tous les actes de la vie civile est soustrait à tout régime de protection " (Cornu, 1987 : 632-633). La condition de mineur est donc associée à une incapacité juridique ou à une capacité juridique limitée : le mineur serait donc titulaire de droits mais ne disposerait pas seul de la possibilité de les exercer, ni de prendre des décisions concernant sa personne ou son patrimoine, ses parents ou les titulaires de l'autorité parentale étant responsables de toutes décisions le concernant (Rosenczveig, 2005 : 1319-1320 ; Lazaro González, 2002 : 42-51).

Les personnes mineures nécessitent donc la protection et l'assistance matérielle, physique et morale de leurs parents. L'absence temporaire ou définitive des adultes initialement désignés pour assumer leur tutelle caractérise la catégorie des mineurs "non accompagnés ", "séparés " ou "isolés ". Comme conséquence de cette absence, on présuppose à juste titre que le mineur se trouvant dans cette situation doit être protégé et assisté par les autorités de l'État où il se trouve. Dans le droit international, la base juridique de cette interprétation se trouve précisée à l'article 20 de la CIDE disposant que " tout enfant qui est temporairement ou définitivement privé de son milieu familial, ou qui

13 Article L221-5 du CESEDA.

14 Sénat (2013) Proposition de Loi $n^{\circ} 154$ relative à l'accueil et à la prise en charge des mineurs isolés étrangers, 20 novembre, Session ordinaire de 2013-2014, 11 p.

15 Ministre de la Justice (2013) Circulaire relative aux modalités de prise en charge des jeunes isolés étrangers : dispositif national de mise à l'abri, d'évaluation et d'orientation, 31 mai, 6 p. 
dans son propre intérêt ne peut être laissé dans ce milieu, a droit à une protection et une aide spéciales de l'État ". En cas de doute, le Comité des Droits de I'Enfant des Nations Unies a confirmé I'applicabilité de ce principe à la situation des enfants non accompagnés et séparés ${ }^{16}$. Au niveau des droits nationaux, la protection de l'enfance s'articule à partir des notions de danger, de délaissement ou d'abandon définies dans les différents droits civils et qui légitiment l'intervention protectrice de l'État ${ }^{17}$.

À la condition de mineurs privés d'assistance et de protection familiale se juxtapose la condition d'étranger. La notion d'étranger se construit sur la base d'un critère de nationalité que chaque État se réserve le pouvoir d'attribuer en exercice de sa souveraineté (Barbou des Places, 2010 : 42) et qui justifie un traitement légal différencié et souvent discriminatoire des non-nationaux. L'étranger en tant qu'individu n'ayant pas la nationalité du pays dans lequel il réside est soumis à une série de restrictions dans l'exercice de ses droits civils, sociaux et politiques par rapport aux nationaux (Julien-Laferrière, 2000 : 14). En règle générale, les droits des personnes non nationales sont limités au niveau législatif ou soumis à condition d'exercice, en particulier en ce qui concerne la possibilité d'accéder, de séjourner et de s'établir sur le territoire, l'accès libre au travail, l'accès aux soins de santé, l'éducation, I'hébergement, etc. Certains autres droits, en particulier le droit de vote, sont habituellement interdits d'exercice pour les étrangers. En revanche, les droits inhérents à toute personne comme le droit à la vie et à la l'intégrité physique, le droit à la liberté idéologique et de culte et le droit d'accès à la justice, sont difficilement modulables par les législateurs nationaux au risque d'entrer en contradiction avec les principes du droit international des Droits de l'Homme universellement reconnus. Or, comme il a été évoqué préalablement, la discrimination législativement tolérée de la personne étrangère dans la reconnaissance et l'exercice de ses droits devrait être pourtant inopérante en ce qui concerne les droits de l'enfant, et cela en application de l'article 2 de la Convention de New York ${ }^{18}$.

Cette impossibilité même partielle de "discriminer " le mineur étranger qu'impose le droit international illustre la complexité et les contradictions que présente le traitement de la catégorie que nous analysons et qui correspond à un découpage plus ou moins objectif d'une réalité empirique (Lochak, 1992 : 291). Le double versant juridique qui la caractérise (mineur privé d'assistance et de protection de ses représentants légaux méritant protection institutionnelle vs personne étrangère susceptible de bénéficier des prestations sociales et de s'intégrer dans le marché du travail) perturbe par son antagonisme l'action des autorités étatiques chargées de leur accueil et de leur prise en charge. Comme nous avons pu le vérifier lors de nos recherches précédentes (Senovilla Hernández, 2010), alors que les mineurs non accompagnés, séparés ou isolés étaient initialement tolérés et accueillis par les autorités de protection de l'enfance, les autorités compétentes en matière d'immigration et d'asile

\footnotetext{
16 Comité des Droits de I’Enfant des Nations Unies (2005) Observation générale $n^{\circ}$ 6, op. cit., paragraphe 39.

17 Article 375 du Code Civil français (situation de danger) ; article 172 du Code Civil espagnol (situation de délaissement) ; article 403 du Code Civil italien (situation d'abandon) ; article 17 du Chidren's Act du Royaume-Uni (situation de nécessité). 18 Voir note 4.
} 
tendaient à restreindre les possibilités d'insertion durable dans les sociétés d'accueil, soit par leur rapatriement vers leur pays d'origine, soit en limitant les possibilités d'obtention d'un statut régulier en tant que personnes étrangères. L'ambivalence des définitions du mineur migrant en situation de danger conduit aujourd'hui à privilégier un traitement juridique centré sur la condition d'étranger. Nos dernières enquêtes de terrain soulignent même que le versant protecteur de l'approche juridique tend à s'amoindrir dans de nombreux pays européens (Senovilla Hernández, 2013).

\section{Des pratiques impliquant l'exclusion de la catégorie}

Les mineurs étrangers constituent un bon exemple des contradictions et des limites que présente l'application des Droits de I'Homme (Masson, 2006 : 607) à une réalité où la souveraineté des États, menant à une discrimination des étrangers, prime sur le cadre juridique international, qui oblige à protéger les enfants en danger car privés de leur entourage familial proche.

La dimension nationale évoquée se caractérise d'abord par une absence de définition législative de la figure du mineur étranger privé de son entourage familial. Elle se distingue ensuite par une interprétation et une application instrumentalisées et sécuritaires des normes existantes et, enfin, par toute une série de pratiques institutionnelles qui - à notre sens - cherchent à dénaturer cette catégorie en minorant les éléments de protection la constituant. Cette affirmation est basée sur les résultats des travaux conduits dans le cadre du projet PUCAFREU qui ont montré une évolution (ou plutôt une régression) dans les pratiques administratives à destination de cette population (Senovilla Hernández, 2013). Depuis leur apparition à la fin des années 1990, on peut affirmer que les mineurs non accompagnés ou isolés ont été majoritairement intégrés dans les systèmes de protection des différents États d'accueil, bien que la qualité de la prise en charge ait pu être contestable et que l'absence de solutions durables ait pu aboutir à leur exclusion à l'approche de la majorité. Plus récemment, et dans un contexte généralisé de crise économique provoquant des réductions budgétaires sévères au sein des services sociaux européens, les autorités de différents pays européens sont davantage tentées par des pratiques visant à contester la reconnaissance de l'appartenance à cette catégorie de migrants et, en conséquence, à filtrer l'accès à la protection et à l'assistance que ces mineurs sont en droit d'attendre. Autrement dit, le problème de la double appartenance juridique du mineur migrant signalé par Masson évolue de facto vers une progressive négation de la condition de mineur en situation de danger propre à cette population et sur laquelle se base le traitement protecteur des États (Masson, 2006 : 8-12).

Le traitement légal des mineurs migrants sans référents parentaux dans les différents contextes nationaux de I'Union européenne s'articule autour de trois étapes clés de leur parcours : leur première identification, le contenu de la prise en charge proposée et la détermination (ou l'indétermination) d'une solution durable. Les pratiques que nous allons décrire tout au long de cette section ont comme conséquence la soumission du mineur étranger aux aléas de l'évaluation plus ou moins bienveillante des agents chargés de représenter les intérêts de l'État ou de ses institutions (Spire, 2010 : 359). Le droit international garant de 
l'intérêt de l'enfant est couramment violé ou mis de côté du fait de son manque de pouvoir effectif et de " l'absence de visibilité des étrangers comme détenteurs de droits " (Chetail, $2012: 23$ ).

\section{L'identification}

Lors de l'ouverture du processus d'identification - qu'il soit réalisé à un poste frontalier ou à l'intérieur d'un territoire -, les mineurs migrants subissent de la part des autorités d'immigration ou de protection de l'enfance une série de pratiques qui sont susceptibles d'entraver leur reconnaissance en tant que mineurs non accompagnés ou isolés et, en conséquence, de ne pas leur permettre de bénéficier d'une prise en charge en tant que mineurs en situation vulnérable. Trois pratiques méritent d'être commentées : les privations de liberté des mineurs migrants à leur arrivée sur le territoire d'un État, la contestation de la minorité et la reconnaissance de la situation d'isolement.

Nos données de terrain indiquent qu'un nombre important de mineurs étrangers (car reconnus en tant que tels par les autorités à un stade postérieur) sont privés de liberté à leur arrivée en Europe et parfois enfermés dans des centres de rétention pour personnes migrantes adultes sans aucune considération initiale de leur condition de mineurs et de leurs besoins de protection. Lors de nos enquêtes récentes en Espagne nous avons rencontré cette situation à plusieurs reprises dans le cas de mineurs arrivés par voie maritime " accompagnés " d'autres migrants adultes (Senovilla Hernández, 2013 :102-103). Mais la privation de liberté des mineurs migrants en danger n'est pas réservée à I'Espagne. La Grèce fut condamnée en 2010 par la Cour Européenne des Droits de l'Homme suite à la détention et à la libération ultérieure sans aucune mesure de prise en charge d'un mineur afghan de quinze ans ; pratique considérée comme traitement inhumain et dégradant violant l'article troisième de la Convention européenne des Droits de I'Homme (CEDH $)^{19}$. Encore plus inhumain et dégradant, le cas de la petite Tabitha qui, âgée de cinq ans au moment des faits, a été privée de liberté pendant deux mois par les autorités belges avant d'être renvoyée en République Démocratique du Congo. Tabitha était accompagnée de son oncle (qui n'était pas son représentant légal) et elle essayait de rejoindre sa mère au Canada. La Belgique fut sévèrement condamnée par la Cour de Strasbourg à la suite de cette affaire ${ }^{20}$.

Qu'il s'agisse de la situation espagnole ou bien d'autres contextes européens comme la France, I'Italie ou l'Allemagne, les privations de liberté associées à la condition de migrants qui leur est assignée constituent un premier obstacle que les mineurs étrangers doivent surmonter pour faire valoir leur condition d'enfants méritant protection. II convient de préciser que la Convention des Droits de l'Enfant n'autorise la privation de liberté des mineurs qu'en dernier ressort (en l'absence d'autres options alternatives) et sur une période de temps la plus courte possible. De même, les personnes mineures privées de liberté doivent être séparées des personnes adultes détenues (sauf si le maintien avec

19 Voir Cour européenne des Droits de I'Homme (2011) Affaire Rahimi c. Grèce, 8687/08, Arrêt, Strasbourg, 5 avril. L'article 3 de la CEDH établit que « Nul ne peut être soumis à la torture ni à des peines ou traitements inhumains ou dégradants ".

20 Voir Cour européenne des Droits de l'Homme (2006) Affaire Mubilanzila Mayeka et Kanini Mitunga c. Belgique, 13178/03, Arrêt, Strasbourg, 12 octobre. 
des adultes est dans leur intérêt) et être assistées pour pouvoir contester toute mesure de privation de liberté ${ }^{21}$. La dimension internationale caractérisant le traitement protecteur de notre catégorie se voit - par le biais de cette pratique répandue dans de nombreux contextes nationaux - clairement bafouée.

Une deuxième pratique généralisée dans le contexte européen est la contestation quasi-systématique de l'âge déclaré par les mineurs. Cette mise en doute du premier élément constitutif de notre catégorie juridique (la minorité d'âge) implique une exclusion définitive des individus qui sont considérés majeurs par les autorités et qui ne pourront donc pas bénéficier de la protection qui leur est dévolue.

Lors de leurs premiers contacts avec les autorités compétentes, les mineurs peuvent se déclarer en tant que tels et même parfois appuyer leur déclaration par la production de documents d'identité divers : passeport, carte d'identité, acte de naissance ou autres. Qu'ils soient détenteurs ou non d'un document d'identité, les mineurs sont soumis par les autorités à un ou plusieurs examens afin de déterminer leur âge. La méthode la plus répandue consiste en une radiographie du poignet gauche du mineur, dont les résultats sont comparés avec une grille de mesure du développement osseux élaborée dans les années 1930 aux États-Unis à partir d'un échantillon de population blanche d'origine caucasienne (Gilsanz et Ratib, $2005: 7)$. Ce test est parfois complété par des examens de développement dentaire et de puberté. Néanmoins, la fiabilité de ce type d'examens - et surtout sa pertinence pour déterminer l'âge d'une population adolescente avec des modes de vie, une alimentation, un environnement et des conditions de développement fortement diversifiées - a été contestée de façon répétée par la communauté scientifique. Selon le rapport d'un groupe de travail de l'Académie Nationale de Médecine Française (sollicitée spécifiquement par le ministère de la Justice sur la question de la détermination de l'âge des mineurs isolés) « la lecture de l'âge osseux par la méthode de Greulich et Pyle universellement utilisée, permet d'apprécier avec une bonne approximation l'âge de développement d'un adolescent en dessous de seize ans. Cette méthode ne permet pas de distinction nette entre seize et dix-huit ans ${ }^{22}$. Si logiquement la plupart des mineurs concernés par une contestation de leur minorité sont ceux qui se trouvent dans la tranche d'âge seize-dix-huit ans, lors de récentes enquêtes de terrain réalisées en France nous avons pu constater que des mineurs dont l'apparence physique " semblait " confirmer leur minorité ont été déclarés majeurs suite à la pratique d'un test osseux et alors qu'ils étaient porteurs de

\footnotetext{
21 Article 37 paragraphes b, c et d de la CIDE : " Les États parties veillent à ce que : b) Nul enfant ne soit privé de liberté de façon illégale ou arbitraire. L'arrestation, la détention ou l'emprisonnement d'un enfant doit être en conformité avec la loi, n'être qu'une mesure de dernier ressort, et être d'une durée aussi brève que possible ; c) Tout enfant privé de liberté soit traité avec humanité et avec le respect dû à la dignité de la personne humaine, et d'une manière tenant compte des besoins des personnes de son âge. En particulier, tout enfant privé de liberté sera séparé des adultes, à moins que I'on estime préférable de ne pas le faire dans I'intérêt supérieur de l'enfant [...] ; d) Les enfants privés de liberté aient le droit d'avoir rapidement accès à l'assistance juridique ou à toute autre assistance appropriée, ainsi que le droit de contester la légalité de leur privation de liberté devant un tribunal ou une autre autorité compétente, indépendante et impartiale, et à ce qu'une décision rapide soit prise en la matière ".
}

22 Académie Nationale de Médecine (2007) 07-01 Sur la fiabilité des examens médicaux visant à déterminer l'âge à des fins judiciaires et la possibilité d'amélioration en la matière pour les mineurs étrangers isolés, Rapport adopté le 12 janvier. 
documents d'identité. Une mineure qui avait quatorze ans au moment de son arrivée en France a même évoqué la surprise des policiers suite à l'obtention de résultats d'examen la déclarant majeure ${ }^{23}$.

À Paris, la première évaluation de l'âge se réalise actuellement à partir d'un ou plusieurs entretiens avec le personnel d'une association en charge de cette évaluation et de la situation d'isolement des personnes se déclarant " mineurs isolés étrangers ". Selon I'ADJIE ${ }^{24}$, ce dispositif parisien nommé PAOMIE ${ }^{25}$ devient un filtre et " un outil d'élimination de jeunes qui devraient bénéficier d'une présomption de minorité ${ }^{26}$. Nos enquêtes, réalisées au cours de I'hiver et du printemps 2012, ont largement confirmé le rôle de filtrage exercé par la PAOMIE (Senovilla Hernández, 2013 : 56-63 ; 100-102). Qui plus est, ce dispositif a établi une sous-catégorisation discrétionnaire qui vise à séparer les " mineurs isolés " en deux groupes : ceux considérés être âgés de moins de seize ans et qui sont envoyés aux services de protection de l'Aide Sociale de l'Enfance pour une prise en charge "classique " et ceux évalués comme étant de plus de seize ans qui sont simplement "mis à l'abri ". Cette pratique qui défend ouvertement l'intérêt de la ville de Paris pour ne pas voir son dispositif de protection " saturé " contredit pourtant le principe de non-discrimination de la Convention des Droits de l'Enfant en établissant un traitement restrictif fondé sur le fait que le mineur soit d'un âge plus ou moins proche de la majorité.

La contestation de l'élément " minorité " de la catégorie que nous analysons produit donc une sous-catégorie (sans existence légale) d'enfants migrants exclus de la protection et en situation d'errance sur le territoire européen. II s'agit des jeunes " aventuriers " si l'on reprend le terme utilisé par Pian (2009: 74) en quête de meilleures opportunités de vie et du "lieu " où pouvoir s'installer durablement. La migrerrance (Sadik, 2009 : 49) induite par des politiques migratoires et d'asile qui pénalisent de nombreux migrants et demandeurs d'asile en Europe, acquiert une dimension nouvelle quand les victimes (et à la fois les acteurs) sont des jeunes en pleine adolescence.

Quand la minorité est reconnue, l'autre élément du processus d'identification, susceptible d'être contesté ou instrumentalisé par les autorités, est celui relatif à "l'isolement " ou à la considération de l'enfant en tant que " non accompagné ", condition qui ouvre l'application du droit de protection de l'enfance pour les mineurs en situation de danger ou de délaissement. Si l'on se réfère aux définitions internationale et nationale de la notion d'isolement, il ressort qu'elle est associée à l'absence des parents ou des personnes responsables de l'autorité parentale. Les cas des mineurs migrants qui sont accueillis informellement par des membres de leur famille élargie (qui ne détiennent pas l'autorité parentale)

\section{Enquête PUCAFREU, notes de terrain du 19 juin 2012.}

24 L'ADJIE est une permanence juridique collective mise en place dans la ville de Paris en 2012 pour les mineurs et jeunes majeurs étrangers.

25 La PAOMIE (Permanence d'accueil et d'orientation des mineurs isolés étrangers), gérée par I'association France Terre d'Asile sous financement de la Direction de I'Action Sociale de l'Enfance et de la Santé du Conseil de Paris, constitue I'unique point d'entrée dans le dispositif de prise en charge des mineurs isolés étrangers à Paris.

26 ADJIE (Aide et Défense des Jeunes Isolés Étrangers) (2013) Permanence d'accueil et $d$ 'orientation des mineurs isolés étrangers (PAOMIE), une moulinette parisienne pour enfants étrangers, document du 25 septembre. 
doivent pouvoir entrer dans cette définition (plus spécifiquement dans celle des " mineurs séparés "), et plus particulièrement pour ceux accueillis par des compatriotes ou amis. En l'absence des parents et des tuteurs, les autorités de protection de l'enfance, que ce soit au niveau administratif ou judiciaire, doivent obligatoirement évaluer les conditions de sécurité et de bien-être dans lesquelles se trouvent ces enfants ainsi que le consentement et la bonne disposition des familles d'accueil : si les membres de la famille ou les personnes en charge de l'accueil sont disposés à assumer l'assistance du mineur migrant et si cet environnement familial est adéquat à sa sécurité et à son développement, les autorités peuvent officialiser l'accueil. Un suivi régulier sera nécessaire afin de constater que les conditions initiales sont maintenues. Pourtant, nos résultats de terrain ont montré que dans une majorité de cas les conditions de vie des mineurs migrants en situation d'accueil familial informel sont loin de répondre à ces attentes : les membres de la famille éloignée n'ont pas toujours les moyens nécessaires à cet accueil, ni parfois une réelle volonté de recevoir ces mineurs, en dehors de quelques jours voire quelques semaines (Senovilla Hernández, 2013 : 64-66 ; 89-92). Certains mineurs sont contraints de travailler informellement pour contribuer financièrement au foyer. D'autres, souvent accueillis par des compatriotes adultes dans le cadre d'un accord préalable avec la famille du mineur, peuvent être amenés à réaliser des activités illégales ou délictuelles pour le remboursement de la dette migratoire dans des situations qui "frôlent " I'exploitation. Néanmoins, ces mineurs se trouvent dans une situation particulièrement compliquée car le fait d'être ou d'avoir été accueillis par un membre de leur famille (voire une autre personne adulte) signifie aux yeux des autorités leur exclusion de facto de la catégorie " mineur isolé " ou " non accompagné " et bloque donc l'accès à la protection qui y est associée. Cela sans tenir compte du danger potentiel de leur situation, ni de leurs besoins immédiats de protection (certains ont pu être expulsés des foyers familiaux et se retrouver à la rue).

\section{Le contenu de la prise en charge}

Si le mineur migrant finit par apporter la preuve de sa condition de mineur et de sa situation d'isolement sur le territoire où il se trouve, il pourra enfin bénéficier de son droit à être protégé. Mais de quelle protection a-t-il besoin ?

Le questionnement autour de la notion de protection a pu prendre différentes orientations. Une tendance lourde, présente dans la quasi-totalité de la littérature, a consisté à définir les mineurs non accompagnés, isolés ou séparés comme des sujets extrêmement vulnérables. Certains auteurs ont contesté cette vulnérabilité et ont plutôt mis en avant la capacité d'autonomie des mineurs migrants. Le paradigme de cette position est fourni par les travaux de Mai qui insiste, en conclusion à ses études sur les mineurs et jeunes majeurs migrants qui se prostituent en Europe, sur la nécessité " d'éviter de considérer la prostitution comme une pathologie et de forcer les jeunes marginaux à choisir entre recevoir un soutien et arrêter leurs activités de survie qui correspondent à leur choix de mode de vie ${ }^{27}$ (Mai, $2010: 87$ ). Aujourd'hui, un nombre croissant de contributions (Orgocka, 2012 ; O'Higgins, 2012 ; Vacchiano et Jimenez, 2012 ;

27 La citation originale en langue anglaise est " it is important to avoid pathologizing sex work and forcing marginalized young people to choose between receiving support and giving up livelihood strategies that are consistent with their chosen lifestyles ". 
Senovilla Hernández, 2013) plaide pour une approche capable de conjuguer les deux aspects : des mineurs vulnérables en raison des épreuves vécues tout au long de leur parcours migratoire et du manque de reconnaissance de leurs droits de la part des autorités étatiques (tant dans leurs pays d'origine que dans les pays de transit et de destination) et qui, dans un même temps, montrent une admirable capacité à faire face aux difficultés et obstacles rencontrés, à s'adapter aux différents contextes et situations, à chercher différentes opportunités de vie, à résister, à survivre et à être autonomes. Vulnérabilité et autonomie sont ainsi placées dans une relation de causalité où la capacité d'action des mineurs se trouverait renforcée par l'absence de protection.

La protection proposée ne tient toutefois pas nécessairement compte de ces capacités et ne se définit pas sur la base d'évaluations individualisées des besoins spécifiques de protection de chaque mineur étranger. Les services de protection de l'enfance des différents pays européens ont tendance à privilégier une prise en charge standardisée basée sur les pratiques de protection traditionnellement utilisées pour la population mineure nationale en situation de danger. L'intérêt supérieur de ces enfants est soumis aux contraintes financières et, plus concrètement, aux capacités d'accueil. En France, les directives données par le ministère de la Justice en accord avec l'Assemblée des Départements ont instauré une distribution territoriale des mineurs isolés dans l'idée de déconcentrer la charge financière de la protection ${ }^{28}$. Une fois de plus, l'intérêt supérieur de ces enfants est soumis au coût financier de la protection et, en fin de compte, à I'intérêt des institutions publiques.

Nos enquêtes de terrain montrent une perception variable de la protection par les mineurs migrants (Senovilla Hernández, 2013 : 73-82). Certains apprécient et arrivent à tirer profit de l'assistance reçue (même si leur avenir demeure incertain). En revanche, beaucoup d'autres se plaignent des normes trop strictes, de l'agressivité du personnel encadrant, des négligences dans la gestion de leurs dossiers administratifs, de la confiscation de leurs documents d'identité, des différences de traitement en fonction des origines nationales. Plutôt que protégés, ils se sentent " maltraités ", ce qui conduit à nouveau beaucoup $d^{\prime}$ 'entre eux vers une situation d'exclusion.

\section{Les solutions durables (ou leur manque)}

Selon le Comité des Droits de l'Enfant des Nations Unies, la définition d'une solution durable permettant de répondre aux besoins de protection des mineurs non accompagnés et séparés, en tenant compte de leurs propres opinions, doit constituer l'objectif ultime de leur prise en charge ${ }^{29}$. II existe aujourd'hui un corpus de recommandations et de directives émanant d'institutions internationales et visant à formaliser le processus de détermination de l'intérêt supérieur de l'enfant ${ }^{30}$. Bien souvent, les autorités nationales méconnaissent ces recommandations. Aucun processus de détermination de l'intérêt supérieur

\footnotetext{
28 Voir note 15.

29 Comité des Droits de l'Enfant des Nations Unies (2005) Observation générale $n^{\circ} 6$, op. cit., paragraphe 79 .
}

30 Nous mettons en exergue les directives du Haut Commissariat des Nations Unies pour les Réfugiés (2008) Principes Directeurs de l'HCR relatifs à la détermination de l'intérêt supérieur de l'enfant, mai. 
des mineurs concernés n'est aujourd'hui entamé par les autorités responsables de leur prise en charge. Seuls certains chanceux (ceux qui répondront à des critères d'appréciation souvent discrétionnaires) auront le privilège d'obtenir un statut administratif durable en termes d'immigration ou d'asile : les " papiers ", tant rêvés. D'autres mineurs seront soumis à un retour forcé dans leur pays d'origine, afin d'être regroupés avec leur famille ou non. Dans la plupart des cas, cette décision ne tient compte ni des possibles risques pour l'intégrité et la sécurité du mineur et de sa famille, ni de leur opinion, ni des possibilités de développement pour l'enfant (entendu comme la possibilité de se soigner, d'aller à l'école, d'avoir un niveau de vie digne). Qui plus est, certaines autorités affirment que ce retour représente la meilleure solution dans l'intérêt supérieur de l'enfant, ce qui constitue en fait une interprétation abusive et instrumentalisée du droit international.

Dans cette perspective, I'absence d'application d'une solution durable, qui demeure la situation la plus répandue en Europe, revient à interpréter l'incapacité des autorités comme une stratégie d'attente. Nul ne peut ignorer que cette inaction institutionnelle a comme conséquence de voir ces jeunes venir s'ajouter au nombre des étrangers adultes en situation irrégulière.

\section{Des situations migratoires " hors normes "}

On a analysé jusqu'ici les éléments qui caractérisent la catégorie juridique des mineurs non accompagnés, séparés ou isolés en situation de migration et ensuite, la déconstruction opérée par les institutions responsables de leur accueil et de leur prise en charge à partir d'une interprétation partielle et intentionnelle des normes existantes.

Jusqu'à aujourd'hui, l'accent a été mis sur la condition de ces mineurs en tant que migrants (ou plus précisément en tant que personnes étrangères) au détriment de leur condition de "mineurs privés de leur entourage familial " justifiant une protection inconditionnelle. En dehors de ce constat déjà bien connu, il est nécessaire de pointer un ensemble de situations observées sur le terrain, qui n'entrent pas dans l'arbitrage légal effectué aux niveaux international et national. Les exemples avec lesquels nous allons illustrer cette réflexion sont loin d'être exhaustifs : les recherches qui portent sur ces situations se trouvent toujours à l'état embryonnaire du fait de leur émergence relativement récente et des difficultés et limites d'ordre méthodologique qu'implique leur analyse. Nous avons déjà évoqué le cas des mineurs qui relèvent de la catégorie " séparés " (qui se trouvent avec un membre de leur famille élargie non responsable) et qui essuient un refus de la part des autorités à l'heure d'assumer leur prise en charge, car considérés comme n'étant pas "non accompagnés " ou "isolés ". À cette situation, nous pouvons ajouter deux autres cas de figure : celui des mineurs qui migrent " accompagnés " de personnes adultes à qui l'autorité parentale a été déléguée par leurs parents suite à un accord coutumier ou informel ; et celui des mineurs qui migrent avec leurs parents ou les rejoignent mais qui se trouvent dans une situation d'abandon du fait d'un exercice limité ou inadéquat de l'autorité parentale. 
Dans le premier cas de figure, les exemples sont relativement nombreux et divers. Parfois la transmission de la responsabilité se faisait dans le but ponctuel de traverser une frontière : cela a été le cas des mineurs roumains qui migraient vers I'Italie par voie terrestre avant I'entrée de leur pays dans I'Union européenne et qui portaient invariablement un document de délégation de l'autorité parentale au nom d'autres voyageurs adultes ou même parfois des chauffeurs de cars qui les transportaient (Save the Children Italia, 2006 : 3). D'autres situations relèvent des accords entre familles dans un but migratoire durable : c'est les cas des mineures marocaines et algériennes "kafalées " décrites par Barraud (2008) ${ }^{31}$. Parfois l'accord a pu se produire sans que soit véritablement défini un objectif migratoire préalable et la mobilité a pu survenir à la suite d'un changement brusque du contexte économique et social du pays. Dans le cas des mineurs sénégalais confiés ${ }^{32}$ par leurs familles aux pêcheurs de Dakar et $d^{\prime}$ autres régions sénégalaises pour apprendre le métier, la sévère diminution des ressources halieutiques qu'a dû affronter ce pays dans les années 2000 a vu la reconversion de ces " tuteurs " en passeurs de migrants clandestins à l'heure où la route des Canaries était en plein essor (période 2005-2008). Certains mineurs arrivés dans cette région insulaire de l'Espagne étaient donc " accompagnés " par des adultes qui étaient en principe responsables d'eux (Robin et Senovilla Hernández, 2010 : 145-149).

Face à ces situations, la question posée est celle relative au fait que ces jeunes puissent se retrouver hors de portée de la catégorie " mineurs non accompagnés " et, en conséquence, se voir privés d'une prise en charge si elle s'avère nécessaire. Ce risque est lié à la définition qu'ont pu donner les Nations Unies et I'Union européenne de la catégorie " mineur non accompagné ". À quelques mots près (voir plus haut) les deux définitions font référence aux mineurs " non pris en charge par un adulte responsable selon la Loi ou la coutume ". Une interprétation stricto sensu de cette définition impliquerait que les adolescents sénégalais confiés aux pécheurs-passeurs adultes n'auraient pas pu être considérés comme "non accompagnés ". En dehors de toutes les difficultés d'ordre pratique et logistique que comporte cette reconnaissance de la délégation coutumière de l'autorité parentale et qui donnent, encore une fois, un ample pouvoir discrétionnaire à l'agent de l'État confronté aux situations décrites, l'interprétation stricte de la définition est susceptible d'exclure certains mineurs en situation de danger de la catégorie.

Le second cas de figure se révèle encore plus complexe même s'il intéresse des mineurs migrant avec leurs parents. Nous ne faisons pas ici référence aux ascendants de personnes étrangères qui malgré leur possible situation précaire exercent convenablement leurs responsabilités en tant que père et mère (bien que ces mineurs soient parfois mis en danger du fait du harcèlement que les agents d'immigration peuvent faire subir à leurs parents), nous pensons

31 La kafala constitue, selon Émilie Barraud, un modèle de substitution à l'adoption, formellement interdite dans nombre de pays musulmans. Dans le texte nous faisons référence en particulier à la kafala notariale concernant les mineurs non abandonnés et de filiation connue. À partir d'un arrangement entre deux familles, validé par un acte notarial, I'enfant circule à l'intérieur du cercle de parenté, souvent pour être transféré dans un pays étranger. Voir Barraud (2008: 133-134).

32 Sur la pratique de " confiage " des enfants et son utilisation en Afrique de I'Ouest, voir Pilon et Vignikin (1996 : 484-485). 
plutôt à certains enfants mineurs dont les parents sont déjà dans une situation d'instabilité personnelle et/ou de précarité sociale qui les empêche d'exercer leurs devoirs parentaux de façon correcte. Dans certaines situations extrêmes, ces parents peuvent même exercer une sorte d'exploitation à l'égard de leurs enfants en les poussant vers la pratique d'actes délictuels ou la mendicité. D'autres mineurs, livrés à eux-mêmes, pratiquent ce type d'activités avec la tolérance de leurs proches. Loin de vouloir décrire une situation qui serait généralisée, nous avons pu approcher cette situation dans nos enquêtes auprès de certains enfants Roms ${ }^{33}$. La complexité tient à la présence des deux parents qui exclut toute possibilité formelle d'accès à la catégorie de " mineur isolé-non accompagné " et à la protection associée, ce qui est logiquement l'interprétation des autorités mais aussi de certaines institutions privées de soutien à l'enfance en danger. II est bien entendu possible de considérer que la situation de ces mineurs relève de la protection générale de l'enfance (et de les associer à la catégorie générale des mineurs nationaux en danger dont les parents sont aussi présents) mais ce cas de figure présente des spécificités qui permettent de le rapprocher de la catégorie des mineurs isolés ou non accompagnés (absences prolongées des parents qui rentrent au pays pour différentes raisons, difficultés accrues d'adaptation à la prise en charge " classique ") ou des mineurs victimes de traite (abandon scolaire forcé par l'entourage, soumission aux mariages précoces, etc.) (Peyroux, 2013 : 170). Certaines associations spécialisées ont adapté leurs catégories et utilisent le terme de " mineurs étrangers en danger " afin d'intégrer ces jeunes dans le cadre de leurs interventions et ce malgré la présence d'une autorité parentale. II nous parait essentiel d'approfondir les recherches existantes sur cette population afin d'évaluer l'éventuelle existence d'une lacune législative susceptible de condamner ces enfants à une situation de délaissement durable non prise en compte institutionnellement.

\section{Conclusion}

Les subterfuges légaux et les pratiques de filtrage décrites dans ce texte, qui conduisent à mettre en doute l'état civil et la situation d'abandon des mineurs dits " non accompagnés " ou "isolés ", produisent une catégorie sociale de jeunes exclus du bénéfice de la protection de l'enfance ainsi que de tout autre droit reconnu aux mineurs de dix-huit ans. Parmi ceux qui obtiennent une protection, nombreux sont les mineurs qui font l'objet d'une application instrumentalisée ou restrictive des normes existantes, autant pour ce qui concerne le contenu même de leur prise en charge (leur hébergement, leur éducation, leur santé, leur accompagnement éducatif, leur représentation légale) que de ce qui relève de la définition d'une solution durable, que ce soit la réunification familiale (qui doit être consentie et dans l'intérêt de l'enfant) ou leur intégration durable dans la société d'accueil. Cette politique engendre une nouvelle catégorie de mineurs " maltraités " qui peuvent se soustraire au contrôle des institutions pour revenir à une situation d'exclusion.

33 Cette situation a pu nous être décrite dans les témoignages apportés par des acteurs associatifs travaillant avec cette population. 
Pour justifier leurs pratiques d'exclusion et de maltraitance, les institutions compétentes allèguent l'existence de faux mineurs, les difficultés d'adaptation aux dispositifs existants et, surtout, le coût financier élevé de la prise en charge dans un contexte de crise économique. "Nous ne pouvons pas accueillir toute la misère du monde " est la phrase que l'on peut souvent entendre de la part de certains décideurs pour justifier la traque à laquelle on soumet parfois cette population. Et, alors que l'enjeu financier ne devrait éthiquement ni légalement être évoqué, l'analyse porte plutôt à considérer que les pratiques qui posent aujourd'hui question sont la conséquence de l'absence d'une réelle volonté institutionnelle pour dépasser l'argument financier et avancer vers une démarche concertée. Les solutions adaptées existent et leur coût n'est pas nécessairement plus élevé que celui que doivent supporter les collectivités locales en hébergeant ces mineurs pendant des mois dans des hôtels.

Outre les mineurs exclus et/ou maltraités, certains autres profils de mineurs restent invisibles des institutions qui considèrent que leur situation ne relève pas de la catégorie " mineurs non accompagnés ou isolés ". II s'agit souvent de mineurs qui sont " mal accompagnés " par des personnes adultes (proches ou non) qui ne disposent ni des moyens ni de la capacité nécessaires pour s'en occuper ou qui les exploitent dans différentes activités lucratives (travail informel, petite délinquance, prostitution, mendicité, etc.). Poursuivre la recherche de terrain s'avère plus que nécessaire pour déterminer les spécificités de ces situations et ainsi pouvoir proposer une réponse adaptée qui pourra impliquer une redéfinition de la catégorie légale qui aujourd’hui les exclut.

Au moment où la Convention Internationale des Droits de l'Enfant s'apprête à célébrer son vingt-cinquième anniversaire, les mineurs migrants sans référents parentaux restent une population vulnérable qui doit être protégée et assistée par les autorités de l'État où ils vivent tout en tenant compte de leur capacité $d^{\prime}$ 'action et d'autonomie - forgée en grande partie au cours de leur expérience migratoire -, qui doit être évaluée et dûment considérée au moment de décider des modalités de leur prise en charge. La double condition que présente cette population en tant que mineurs vulnérables et migrants autonomes engendre une polarisation des perceptions de ce phénomène migratoire : victimes pour les uns, profiteurs pour les autres. Les instruments de droit international des Droits de I'Homme mettent l'accent sur leur protection pendant qu'au sein des législations et pratiques nationales leur condition d'étrangers l'emporte sur celle de mineurs en danger. Il reste néanmoins important de souligner que vingt ans après leur émergence sur la scène migratoire européenne, les différents acteurs politiques, institutionnels, associatifs et universitaires continuent à alimenter cette dichotomie de points de vue sans arriver à trouver des solutions satisfaisantes. Les mineurs et jeunes migrants concernés doivent être invités à participer au débat et à la construction d'une réponse tant législative qu'éducative et sociale spécifique à leur condition migratoire tout en respectant leurs droits en tant qu'êtres humains et enfants. 


\section{Références bibliographiques}

Barbou des Places Ségolène (2010) Les étrangers "saisis " par le droit : enjeux de l'édification des catégories juridiques de migrants, Migrations Société, 22 (128), pp. 35-49.

Barraud Émilie (2008) Les multiples usages sociaux de la Kafala en situation de migration : protection et non protection des mineurs recueillis, e-migrinter, 2 , pp. 133-143.

Bhabha Jacqueline (2010) Too much disappointing: the quest for protection by unaccompanied migrant children outside Europe, in Jyothi Kanics, Daniel Senovilla Hernández and KristinaTouzenis Eds., Migrating alone: Unaccompanied and separated children's migration to Europe, Paris, UNESCO publishing, pp. 91-103.

Bhabha Jacqueline (2007) “Un vide juridique? Migrant Children: The Rights and Wrongs, in Carol Bellamy and Jean Zermatten Eds., Realizing the rights of the child, Zurich, Rüffer \& Rub, pp. 206-219.

Bousta Rhita (2010) Essai sur la notion de bonne administration en Droit public, Paris, L'Harmattan, 568 p.

Chetail Vincent (2012) Droit international des migrations : fondements et limites du multilatéralisme, in Habib Ghérari et Rostane Mehdi Dir., La société internationale face aux défis migratoires, Paris, Pedone, pp. 23-69.

Cornu Gérard (1987) Vocabulaire juridique, Paris, Presses Universitaires de France, $1093 \mathrm{p}$.

Diop Marie (2009) Unaccompanied minors' rights within the European Union: Is the EU asylum and Immigration legislation in line with the Convention on the Rights of the Child?, Odysseus Network-Université Libre de Bruxelles, 120 p.

Farrugia Ruth and Touzenis Kristina (2010) The international protection of unaccompanied and separated migrant and asylum-seeking children in Europe, in Jyothi Kanics, Daniel Senovilla Hernández and Kristina Touzenis Eds., Migrating alone: Unaccompanied and separated children's migration to Europe, Paris, UNESCO publishing, pp. 21-55.

Gilsanz Vicente and Ratib Osman (2005) Hand bone age: A digital atlas of skeletal maturity, Springer, $102 \mathrm{p}$.

Julien-Laferrière François (2000) Droit des étrangers, Paris, Presses Universitaires de France, $552 \mathrm{p}$.

Lazaro González Isabel (2002) Los menores en el derecho español, Madrid, Tecnos, 729 p.

Lochak Danièle (1992) La race : une catégorie juridique?, Mots, 33, pp. 291-303.

Mai Nick (2010) Marginalized youn (male) migrants in the European Union, in Jyothi Kanics, Daniel Senovilla Hernández and Kristina Touzenis Eds., Migrating alone: Unaccompanied and separated children's migration to Europe, Paris, UNESCO publishing, pp. 69-89.

Masson Bénédicte (2010) Mineurs isolés étrangers : le sens d'une appellation, Migrations Société, 22 (129-130), pp. 115-128. 
Masson Bénédicte (2006) Le mineur étranger en droit français et européen (Étude sur les droits du mineur étranger), Faculté Jean Monnet, Université Paris-Sud (XI), thèse doctorale dactylographiée.

O'Higgins Aoife (2012) Vulnerability and agency: Beyond an irreconcilable dichotomy for Social Services providers working with young refugees in the UK, in Aida Orgocka and Christina Clark-Kazak, Independent child migrations: Insights into agency, vulnerability and structure, Wiley Periodicals, pp. 79-91.

Orgocka Aida (2012) Vulnerable yet agentic: Independent child migrants and opportunity structures, in Aida Orgocka and Christina Clark-Kazak, Independent child migrations: Insights into agency, vulnerability and structure, Wiley Periodicals, pp. 1-11.

Peyroux Olivier (2013) Délinquants et victimes. La traite des enfants d'Europe de I'Est en France, Paris, Non Lieu, 203 p.

Pian Anaïk (2009) Aux nouvelles frontières de l'Europe. L'aventure incertaine des sénégalais au Maroc, Paris, La Dispute, 237 p.

Pilon Marc et Kokou Vignikin (1996) Stratégies face à la crise et changements dans les structures familiales, in Jean Coussy et Jacques Vallin, Crise et population en Afrique. Crises économiques, politiques d'ajustement et dynamiques démographiques, Paris, CEPED, pp. 471-493.

Rosençzveig Jean-Pierre (2005) Le dispositif français de protection de l'enfance, Paris, Éditions Jeunesse et droit, 1483 p.

Sadik Gérard (2009) Dublin II : I'asile en orbite, in Olivier Clochard, Atlas des migrants en Europe, Paris, Armand Colin, pp. 49-52.

Save the Children Italia (2006) In viaggio verso quale futuro? Minori stranieri non accompagnatti, fra integrazione e devianza, Roma, Save the Children, $9 \mathrm{p}$.

Sayad Abdelmalek (1999) La double absence. Des illusions de l'émigré aux souffrances de l'immigré, Paris, Seuil, $437 \mathrm{p}$.

Senovilla Hernández Daniel (2013) Mineurs isolés étrangers et sans protection en Europe. Projet PUCAFREU. Rapport comparatif final, Poitiers, MIGRINTERCNRS-Université de Poitiers, $128 \mathrm{p}$.

Senovilla Hernández Daniel (2010-a), Mineurs étrangers non accompagnés et séparés en Europe : une analyse comparative de l'application de la Convention Internationale des Droits de I'Enfant dans six pays, Migrations Société, 22 (129130), pp. 99-114.

Senovilla Hernández Daniel (2010-b) Los menores extranjeros no acompañados en Europa, Murcia, Fundación Diagrama, 303 p.

Spire Alexis (2010) Exister juridiquement sans être sujet de droit, in Actualité de la pensée d'Ábdelmalek Sayad: actes du colloque international, 15-16 juin 2006, Casablanca, Éditions Le Fennec, pp. 355-373.

Suárez Navaz Liliana (2004) Niños entre fronteras: migración de menores no acompañados en el Mediterráneo Occidental, Migraciones y desarrollo, 2, pp. 35-48.

Vacchiano Francesco and Jiménez Mercedes (2012) Between agency and repression: Moroccan children on the edge, Children's Geographies, 10 (4), pp. 457-471. 


\section{Daniel Senovilla Hernández}

\section{... Analyse d'une catégorie juridique récente : le mineur étranger non accompagné, séparé ou isolé}

Cette contribution cherche à analyser une nouvelle catégorie de migrants qui a émergé progressivement dans le contexte européen au cours des années 1990. L'article examine d'abord les différents termes et définitions existants à l'échelle internationale et les trois dimensions qui les composent : la minorité d'âge, l'extranéité et l'isolement représenté par l'absence temporaire ou définitive des parents ou tuteurs du mineur. L'analyse de la catégorie juridique est ensuite mise en contraste avec les données de terrain recueillies lors d'une enquête menée dans quatre États de l'Union européenne entre 2011 et 2012. L'étude des pratiques observées au sein de ces États souligne une multiplication des refus d'appartenance à la catégorie " mineur non accompagné " ou " mineur isolé ". Pour les mineurs, ce durcissement se traduit par leur exclusion de la protection étatique à laquelle ils ont droit selon les normes internationales. Enfin, l'article introduit une réflexion sur les limites de cette catégorie à partir de situations constatées au cours de cette enquête et qui restent à ce jour en marge des définitions existantes.

\section{... Analysing a Recent Legal Category: Unaccompanied or Separated Children}

The aim of this contribution is to analyse a new category of migrants - unaccompanied minors - that emerged progressively within the European context during the 1990 's. Firstly, the paper examines the different terms and definitions existing on an international scale as well as the three elements defining this category: Minority of age, alien-ship, and the fact of being temporarily or permanently deprived of their parents or legal guardians. Secondly, the analysis of the legal category is contrasted with fieldwork results collected during a cross-national survey in four European Union Member States between 2011 and 2012. The findings of this research show how European authorities' practices evolve towards a progressive dispute of the candidates' actual affiliation to the "unaccompanied minor" category. In practice, this means that potential unaccompanied children are excluded from State protection and assistance in contradiction with what International Law mandates. Finally, the article addresses the potential gaps of the category by noting a number of situations observed during fieldwork, which are not included within current definitions.

\section{... Análisis de una categoría jurídica reciente: el menor extranjero no acompañado o separado}

El objetivo de este artículo es analizar una nueva categoría jurídica - el menor extranjero no acompañado - que surgió progresivamente en el contexto europeo durante los años 1990. El texto examina en primer lugar los términos y definiciones existentes a escala internacional así como los elementos que componen la categoría: la minoría de edad, la condición de extranjero y la situación de privación temporal o definitiva de los titulares de la patria potestad. En segundo lugar, el análisis de la categoría jurídica es contrastado con los resultados de una investigación de campo llevada a cabo en cuatro países de la Unión europea entre 2011 y 2012. Tales resultados muestran que las prácticas de las autoridades europeas responsables de esta población evolucionan hacia una progresiva contestación de la pertenencia a la categoría, lo cual conlleva como consecuencia la exclusión de numerosos menores no acompañados de la protección y asistencia a la que tienen derecho en virtud de los postulados del derecho internacional. Finalmente el artículo aborda las posibles carencias y lagunas de la categoría señalando una serie de situaciones constatadas durante el trabajo de campo que no están previstas en las definiciones actuales. 